\title{
Influência dos parâmetros de processamento e sinterização sobre o controle dimensional relativo de cerâmicas à base de alumina com geometria hemisférica
}

\author{
(Influence of processing and sintering parameters on \\ the relative dimensional control of alumina ceramics \\ with hemispheric geometry)
}

\author{
C. D. Rocha ${ }^{1,2}$, M. A. P. Santos ${ }^{1}$, J. M. Melo ${ }^{1}$, A. R. C. Souza ${ }^{2}$, C. A. Costa Neto ${ }^{3}$ \\ ${ }^{I}$ Grupo de Materiais, Instituto de Pesquisas da Marinha - IPqM, R. Ipiru s/n, Cacuia, Ilha do Governador, \\ Rio de Janeiro, $R J$ \\ ${ }^{2}$ Universidade do Estado do Rio de Janeiro - UERJ, R. S. Francisco Xavier 524, Maracanã, Rio de Janeiro, RJ \\ ${ }^{3}$ Universidade Federal do Rio de Janeiro - UFRJ, Programa de Engenharia Metalúrgica e de Materiais, \\ (PEMM/COPPE), Centro de Tecnologia, Bl. F/210, Rio de Janeiro, RJ \\ cassiano@ipqm.mar.mil.br, cida@ipqm.mar.mil.br,jairmmel@yahoo.com.br,a.rayboltt@gmail.com,celio@ \\ metalmat.ufrj.br
}

\section{Resumo}

\begin{abstract}
Na produção de componentes cerâmicos, além de se buscar propriedades especificas e superiores, também se busca produzir peças com dimensões que atendam aos requisitos do projeto. Um dos exemplos da importância do controle dimensional é a produção de cerâmicas do tipo piezoelétricas à base de titanato-zirconato de chumbo, aplicadas em transdutores hidroacústicos, cujo processamento não é trivial, sendo dispendioso e de risco ao manuseio (por causa da presença de chumbo), principalmente se a geometria especificada for hemisférica. Para a otimização do processamento (conformação e sinterização) destas cerâmicas pode-se utilizar outros insumos, como alumina, menos agressivo ao meio ambiente, maior disponibilidade, maior facilidade para processamento, de baixo risco ao manuseio, bem como, de baixo custo. Por esta razão, neste trabalho foram utilizadas duas composições à base de alumina, $\mathrm{A}\left(96,1 \% \mathrm{Al}_{2} \mathrm{O}_{3}, 3,0 \% \mathrm{SiO}_{2}, 0,9 \% \mathrm{MgO}\right)$ e $\mathrm{B}\left(99,1 \% \mathrm{Al}_{2} \mathrm{O}_{3}, 0,9 \% \mathrm{MgO}\right)$ e temperaturas de sinterização de $1550{ }^{\circ} \mathrm{C}$ e $1650{ }^{\circ} \mathrm{C}$. O objetivo deste estudo foi produzir uma cerâmica com geometria hemisférica, de alta densidade, com controle dimensional relativo, por meio de prensagem uniaxial para conformação. Observou-se que a melhor relação entre o controle dimensional e a alta densificação foi obtida com a composição A e sinterização a $1550{ }^{\circ} \mathrm{C}$, em presença de fase líquida pela adição de $\mathrm{SiO}_{2}$. Apesar de os resultados para a composição $\mathrm{B}$ com sinterização a $1650{ }^{\circ} \mathrm{C}$ terem sido aparentemente melhores, esta composição teve sua densificação comprometida e muito abaixo da esperada. Com os resultados obtidos da relação entre controle dimensional e processamento, podem-se aplicar algumas das etapas utilizadas para a produção de cerâmicas à base de PZT com alta densidade, por uma técnica de conformação simples, e geometria complexa (hemisférica), exigida como requisito de projeto de transdutores hidroacústicos.

Palavras-chave: processamento cerâmico, geometrias complexas, controle dimensional.
\end{abstract}

\begin{abstract}
In the production of ceramic components, specific and better properties are required to produce pieces according to the project. One example of the importance of the dimensional control is the production of piezoelectric ceramic for hydroacoustic transducers. Its processing is not trivial, is expensive and risky to handle (due to the presence of lead), particularly for hemispheric shape. For the optimization of the processing (mechanical consolidation and sintering) of these ceramics, other materials may be used, such as alumina, less aggressive to the environment, with higher availability, easier processing, low risk for handling, as well as low cost. In this work two compositions based on alumina were used, $\mathrm{A}\left(96.1 \% \mathrm{Al} \mathrm{O}_{3}, 3.0 \%\right.$ $\left.\mathrm{SiO}_{2}, 0.9 \% \mathrm{MgO}\right)$ and $\mathrm{B}\left(99.1 \% \mathrm{Al}_{2} \mathrm{O}_{3}, 0.9 \% \mathrm{MgO}\right)$, and two sintering temperatures, $1550{ }^{\circ} \mathrm{C}$ and $1650{ }^{\circ} \mathrm{C}$. The objective of this study was to produce a ceramic with complex hemispherical geometry, high density and relative dimensional control, consolidated by dry pressing. It was observed that the best ratio of dimensional control and high densification was achieved with the A composition and sintering temperature $1550{ }^{\circ} \mathrm{C}$, by liquid phase formed by adding $\mathrm{SiO}_{2}$. Although the results for composition $B$ sintered at $1650^{\circ} \mathrm{C}$ were apparently the best, this composition had densification much lower than expected. With the results of the relationship between dimensional control and processing can carry some of the steps used for the production of PZT-based ceramics with high density, by a simple mechanical consolidation and complex geometry (hemispherical) required for hydro-acoustic transducers.
\end{abstract}

Keywords: ceramics processing, complex geometry, dimensional control. 


\section{INTRODUÇÃO}

Os materiais cerâmicos avançados vêm se destacando como material de engenharia no projeto e elaboração de equipamentos de alta tecnologia. Dentre as várias classes de cerâmicas avançadas, são encontradas as cerâmicas eletrônicas que apresentam como uma das características o efeito piezoelétrico sendo denominadas cerâmicas piezoelétricas, cujas propriedades encontram aplicação em setores da indústria como eletrônica, comunicações, aeroespacial, defesa, automobilística, nuclear, biomédica, entre outras [1]. Além disso, quanto maior o nível tecnológico de aplicação destas cerâmicas, maiores serão os custos de produção, bem como as dificuldades para seu processamento, devido às características das matérias-primas iniciais, além da geometria, muitas vezes complexa. Uma das matérias-primas mais utilizadas para a produção de cerâmicas piezoelétricas é o titanato-zirconato de chumbo (PZT) que apresenta como um dos principais limitantes para sua conformação e sinterização a geração de resíduos de chumbo, constituinte importante para o efeito piezoelétrico [1], porém, extremamente agressivo ao meio ambiente e aos trabalhadores que produzem ou manuseiam estes materiais. Para que as propriedades piezoelétricas sejam maximizadas devido à necessidade recorrente de seu emprego, as cerâmicas à base de PZT, além de alta densidade, podem ter como requisito primordial uma geometria específica. Entre elas, podemos citar a forma hemisférica, com aplicação em transdutores em dispositivos biomédicos, sistemas de armas, e estações de força. Portanto, para a produção de cerâmicas à base de PZT, tendo como premissa a geometria a ser obtida, temos duas opções, a otimização do processamento de forma a gerar o menor impacto possível, ou buscar novos materiais que não utilizem compostos tão agressivos e de elevado risco [2]. Matérias-primas alternativas podem ser utilizadas para simular o estudo de parâmetros e etapas de processamento para a produção de cerâmicas de geometria complexa. Entre elas podemos citar a alumina, material de engenharia menos dispendioso e de processamento consolidado e sua utilização, em tempos atuais, abrange os mais diversos ramos da engenharia e ciência dos materiais, como em espumas cerâmicas para a indústria do petróleo [3], agentes ligantes em materiais refratários [4]. Alumina e PZT podem se relacionar por etapas de conformação (prensagem uniaxial) e sinterização, bem como preparo (secagem e homogeneização) e técnicas de caracterização (densidade) de suas matériasprimas, corpos-de-prova a verde e sinterizados.

O objetivo deste trabalho foi estudar a relação entre os parâmetros de processamento e sinterização com o controle dimensional relativo para produção de cerâmicas à base de alumina com geometria complexa, em formato hemisférico, utilizando a prensagem uniaxial de ação simples como técnica de conformação.

\section{MATERIAIS E MÉTODOS}

Foram empregadas como matérias-primas alumina $\left(\mathrm{Al}_{2} \mathrm{O}_{3}\right.$, Alcoa, Brasil), sílica $\left(\mathrm{SiO}_{2}\right)$ e óxido de magnésio ( $\mathrm{MgO}$,
Montedison Farm. Carlo Erba), como recebidas. Foram preparadas duas composições, $\mathrm{A}\left(96,1 \% \mathrm{Al}_{2} \mathrm{O}_{3}, 3,0 \% \mathrm{SiO}_{2}\right.$, $0,9 \% \mathrm{MgO})$ e $\mathrm{B}\left(99,1 \% \mathrm{Al}_{2} \mathrm{O}_{3}, 0,9 \% \mathrm{MgO}\right)$ [5]. Ambas foram homogeneizadas em moinho de rolos por $24 \mathrm{~h}$, em meio aquoso, com adição de $3 \%$ em peso de álcool polivinílico como ligante. Após a homogeneização, as composições foram submetidas a duas etapas de secagem a fim de evitar segregação entre partículas maiores e menores no pó final: a primeira em placa aquecedora a $70^{\circ} \mathrm{C}$ com agitação por $2 \mathrm{~h}$ e a segunda em estufa a $70^{\circ} \mathrm{C}$ por $24 \mathrm{~h}$. Em seguida as composições foram desaglomeradas e peneiradas em peneiras de 30 e 60 mesh. As composições foram colocadas em molde de aço SAE 1045, juntamente com desmoldante, e conformadas com pressão de compactação $60 \mathrm{MPa}$ em prensa uniaxial pela técnica de ação simples. O processo de sinterização foi em forno convencional até $1700{ }^{\circ} \mathrm{C}$ de acordo com os ciclos térmicos I e II. O ciclo térmico I com taxa de aquecimento $2{ }^{\circ} \mathrm{C} / \mathrm{min}$ até $400{ }^{\circ} \mathrm{C} / 2 \mathrm{~h}$, visando à retirada lenta e completa do ligante. Em seguida, uma taxa de aquecimento $4{ }^{\circ} \mathrm{C} / \mathrm{min}$ até $1650{ }^{\circ} \mathrm{C} / 2 \mathrm{~h}$ (temperatura de sinterização), com resfriamento a $4{ }^{\circ} \mathrm{C} / \mathrm{min}$ até $70{ }^{\circ} \mathrm{C}$. $\mathrm{O}$ ciclo térmico II e igual ao ciclo térmico $\mathrm{I}$, porém com temperatura de sinterização $1550^{\circ} \mathrm{C}$.

As caracterizações foram feitas nas matérias-primas, composições A e B, nas peças conformadas a verde e nas peças sinterizadas. Para as matérias-primas foram determinadas as densidades pela técnica de picnometria de hélio a fim de calcular, pela regra das misturas inversa, as densidades das composições A e B. Foram avaliadas as fases cristalinas por difração de raios $\mathrm{X}$. As peças conformadas a verde foram caracterizadas quanto a densidade a verde, pelo método geométrico, e as dimensões (diâmetros interno $\left(\mathrm{D}_{\mathrm{i}}\right)$ e externo $\left(\mathrm{D}_{\mathrm{e}}\right)$ e alturas interna $\left(\mathrm{h}_{\mathrm{i}}\right)$ e externa $\left.\left(h_{e}\right)\right)$, por medição de coordenadas, Fig. 1. As peças sinterizadas foram caracterizadas quanto às fases cristalinas por difração de raios $\mathrm{X}$, formadas devido aos ciclos térmicos, densidade e porosidade pela técnica de Arquimedes, dimensões por medição de coordenadas, Fig. 1, e módulo de Young por inspeção ultrassônica.

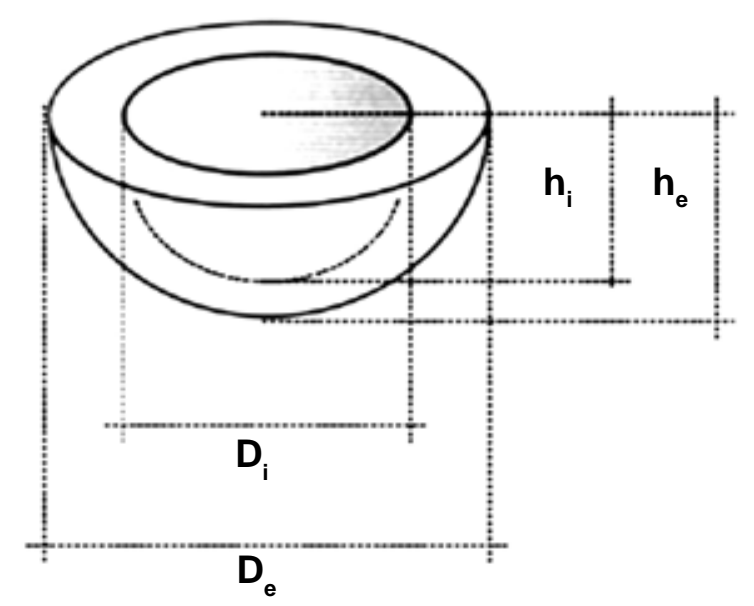

Figura 1: Dimensões aferidas.

[Figure 1: Measured dimensions.] 


\section{RESULTADOS E DISCUSSÃO}

As densidades das matérias-primas $\mathrm{Al}_{2} \mathrm{O}_{3}, \mathrm{SiO}_{2}$ e $\mathrm{MgO}$ foram avaliadas, Tabela I.

Com os dados da Tabela I e pela regra das misturas inversa, equação A, na qual a densidade da mistura $(\rho)$ pode ser calculada pela relação entre a fração mássica de cada componente $\left(\mathrm{w}_{\mathrm{i}}\right)$ e sua densidade $\left(\rho_{\mathrm{i}}\right)$. Os resultados para a densidade das composições A e B estão na Tabela II

Tabela I - Densidade das matérias-primas obtidas por picnometria a hélio.

[Table I - Density of raw materials obtained by helium picnometry.]

\begin{tabular}{cc}
\hline Matérias-primas & Densidade $\left(\mathrm{g} / \mathrm{cm}^{3}\right)$ \\
\hline $\mathrm{Al}_{2} \mathrm{O}_{3}$ & 3,94 \\
$\mathrm{SiO}_{2}$ & 2,12 \\
$\mathrm{MgO}$ & 2,62 \\
\hline
\end{tabular}

$$
\rho=1 / \Sigma\left(w_{\mathrm{i}} / \rho_{\mathrm{i}}\right)
$$

Em relação ao tamanho médio de partículas, observouse que a composição $\mathrm{A}$, cujos valores para o diâmetro são em média maiores, teve maior densificação. Este resultado pode ser atribuído à presença da fase líquida formada por $\mathrm{SiO}_{2}$ que se sobrepôs ao efeito do tamanho de partículas das composições. Para as peças a verde e sinterizadas submetidas aos ciclos térmicos propostos, os resultados de densidade, em termos percentuais, estão na Tabela III.

A caracterização dos processos de sinterização, em estado sólido e em presença de fase líquida, pode ser verificada, indiretamente, pela observação das densidades das peças ao longo do processamento, ou seja, desde a peça verde até a sinterizada, em relação às variáveis do processo como, por exemplo, composição e temperatura [6]. As cerâmicas produzidas com a composição A e para o ciclo térmico I apresentaram as maiores densidades relativas, devido a formação de fase líquida pelo $\mathrm{SiO}_{2}$ em altas temperaturas. Este favorecimento se deve ao fato da fase líquida transportar material pelo interior da microestrutura da cerâmica, preenchendo os poros ou a região dos pescoços, tornando a cerâmica mais densa [7]. Além disso, como o processo de sinterização é um processo termicamente ativado [8], podemos esperar maior densificação para as maiores temperaturas.

As fases cristalinas das composições A e B e formadas após ciclos térmicos a $1550^{\circ} \mathrm{C} \mathrm{e} 1650^{\circ} \mathrm{C}$ estão representadas no difratograma da Fig. 2.

A fase $\alpha-\mathrm{Al}_{2} \mathrm{O}_{3}$ foi identificada como predominante para as composições A e B. Não foram identificados $\mathrm{MgO}$, devido a quantidade adicionada nas composições estar abaixo do limite de detecção do equipamento, bem como $\mathrm{SiO}_{2}$ por ser amorfo, e também não foram identificadas outras fases ou contaminantes. Após o processo de sinterização, as fases cristalinas foram identificadas como predominante a $\alpha-\mathrm{Al}_{2} \mathrm{O}_{3}$, independente da composição ou da temperatura de sinterização, devido ao alto teor de alumina em ambas as composições. Porém, para as peças produzidas com a composição B foi identificada, também, uma segunda fase, o espinélio, como previsto pelo diagrama de fases [9].

Os resultados para o módulo de Young e porosidade estão na Tabela IV.

O controle das mudanças de velocidade ultrassônica, durante a etapa de sinterização, pode servir como um método indireto de determinação do processo de densificação [10]. Além disso, quanto maior for a densificação do material submetido a inspeção ultrassônica, com maior velocidade

Tabela II - Densidade e tamanhos médios de partículas $\left(\mathrm{d}_{50}\right)$ para as composições A e B. [Table II - Densities and average particle size $\left(d_{50}\right)$ for compositions A and B.]

\begin{tabular}{lcc}
\hline \multicolumn{1}{c}{ Composições } & $\begin{array}{c}\text { Densidade } \\
\left(\mathrm{g} / \mathrm{cm}^{3}\right)\end{array}$ & $\begin{array}{c}\text { Tamanho médio de } \\
\text { partícula } \\
(\mu \mathrm{m})\end{array}$ \\
\hline $\mathrm{A}\left(96,1 \% \mathrm{Al}_{2} \mathrm{O}_{3} / 3,0 \% \mathrm{SiO}_{2} / 0,9 \% \mathrm{MgO}\right)$ & 3,82 & 4,16 \\
$\mathrm{~B}\left(99,1 \% \mathrm{Al}_{2} \mathrm{O}_{3} / 0,9 \% \mathrm{MgO}\right)$ & 3,92 & 3,47 \\
\hline
\end{tabular}

Tabela III - Densidades das peças cerâmicas a verde e sinterizadas (submetidas aos ciclos térmicos I e II) para as composições A e B.

[Table III - Density of the green and sintered ceramic (subjected to thermal cycling I and II) for compositions $A$ and B.]

\begin{tabular}{cccc}
\hline \multirow{2}{*}{ Composição } & Densidade & \multicolumn{2}{c}{ Densidade (\%) } \\
\cline { 3 - 4 } & a verde $(\%)$ & $1650^{\circ} \mathrm{C}$ & $1550{ }^{\circ} \mathrm{C}$ \\
\hline A & $55,92 \pm 0,62$ & $96,44 \pm 0,15$ & $91,05 \pm 0,73$ \\
B & $55,74 \pm 0,63$ & $82,31 \pm 0,56$ & $68,23 \pm 1,10$ \\
\hline
\end{tabular}



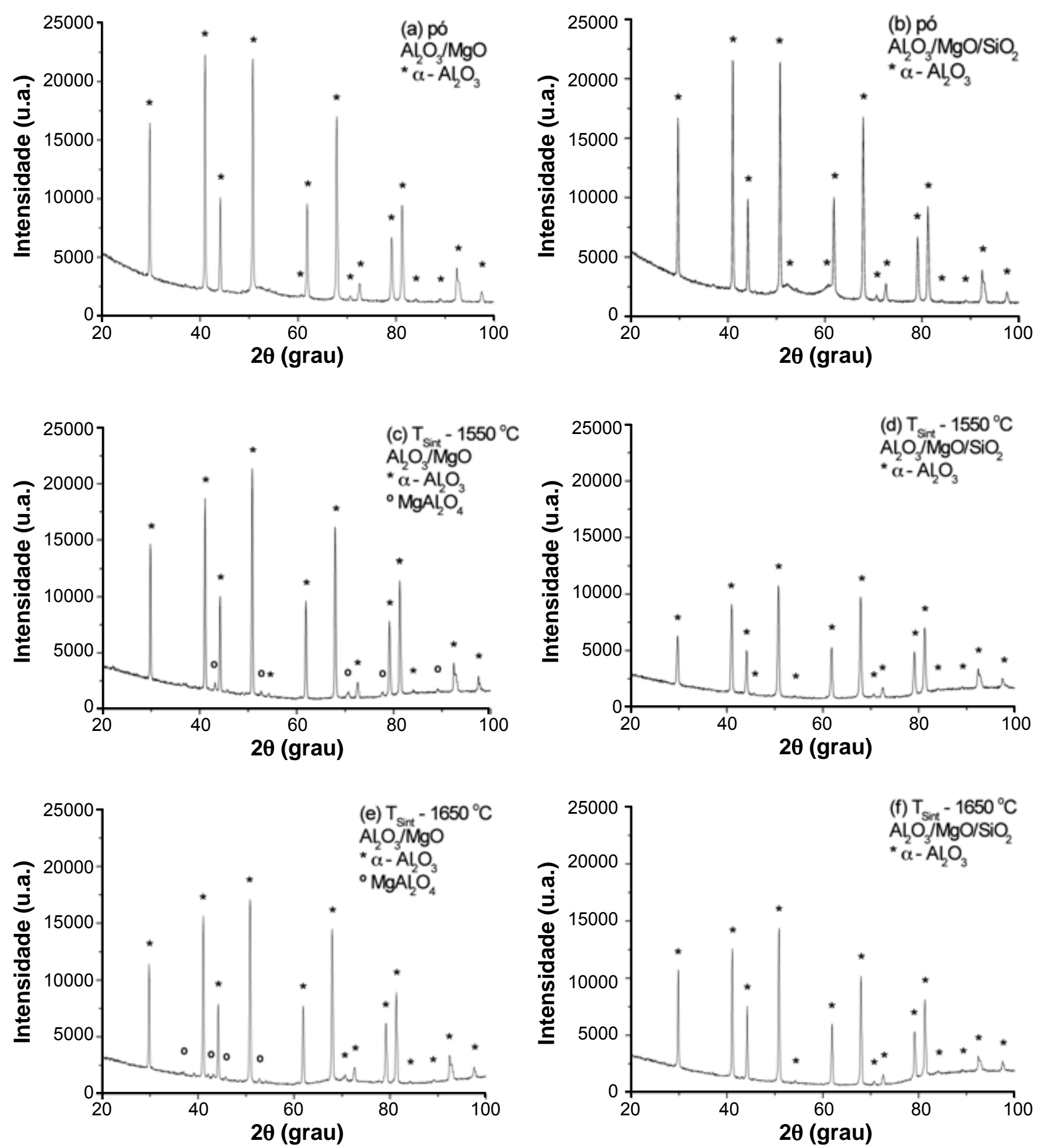

Figura 2: Difratogramas de raios $\mathrm{X}$ das composições A e B e das peças sinterizadas a $1550{ }^{\circ} \mathrm{C}$ e $1650{ }^{\circ} \mathrm{C}$.

[Figure 2: X-ray diffraction patterns of compositions $A$ and $B$ sintered at $1550^{\circ} \mathrm{C}$ and $1650^{\circ} \mathrm{C}$.]

se propagará a onda sonora, e portanto, esta velocidade, também, é um indicativo da isotropia do material [11]. As cerâmicas produzidas pela composição A apresentaram os maiores módulos de Young, pois, as velocidades tranversal e longitudinal, se propagam melhor quanto maior for a densidade [11], e conseqüentemente, menor for a porosidade do material em estudo, conforme observação da Tabela IV e Fig. 3.

Como ambas as composições apresentam um percentual de alumina superior a $95 \%$ e os resultados de difração de 
Tabela IV - Porosidade relativa e módulo de Young das peças sinterizadas de composições A e B, submetidas aos ciclos térmicos I e II.

[Table IV - Relative porosity and Young's modulus of sintered parts of compositions $A$ and $B$, subjected to thermal cycling I and II.]

\begin{tabular}{ccccc}
\hline Composição & \multicolumn{2}{c}{$\begin{array}{c}\text { Porosidade relativa } \\
(\%)\end{array}$} & \multicolumn{2}{c}{$\begin{array}{c}\text { Módulo de Young } \\
(\mathrm{GPa})\end{array}$} \\
\hline & $1650{ }^{\circ} \mathrm{C}$ & $1550^{\circ} \mathrm{C}$ & $1650{ }^{\circ} \mathrm{C}$ & $1550{ }^{\circ} \mathrm{C}$ \\
\hline $\mathrm{A}$ & 0,09 & 0,92 & 321,37 & 266,18 \\
$\mathrm{~B}$ & 10,41 & 23,88 & 216,29 & 97,80 \\
\hline
\end{tabular}

raios $\mathrm{X}$ terem como fase predominante $\alpha-\mathrm{Al}_{2} \mathrm{O}_{3}$, podemos comparar os módulos de Young obtidos experimentalmente com equações empíricas, equações $\mathrm{B}, \mathrm{C}$ e D $[12,13]$, observando as especificidades e propriedades de cada material estudado. Para isto foi avaliada a tendência com a qual os dados se comportaram em relação à porosidade (P), Fig. 4, na qual $\mathrm{E}_{0}$ é o módulo de Young para peças com porosidade zero e $\mathrm{b}$ é uma constante, 1,96 .

$$
\begin{aligned}
& \mathrm{E}=\mathrm{E}_{0}\left(1-\mathrm{b}^{* P}\right) \\
& \mathrm{E}=\mathrm{E}_{0}\left[1-\mathrm{b}^{* P}+(\mathrm{b}-1)^{*} \mathrm{P}^{2}\right] \\
& \mathrm{E}=\mathrm{E}_{0} \exp (-\mathrm{b} * \mathrm{P})
\end{aligned}
$$

A equação B é uma aproximação para o modelo de Voigt (supondo $b=1$ ) [14], na qual foi admitido que uma das fases é a porosidade, com módulo de Young atribuído como zero, e que a deformação em ambas as fases é igual. A equação $C$ é uma aproximação da equação reportada em [14], com b igual a 1,9, considerando uma razão de Poisson 0,3 e porosidade inferior a $50 \%$. Já a equação $\mathrm{D}$ foi proposta em [15].

Na Fig. 4, em todos os modelos propostos, a tendência do módulo de Young é decrescer com o aumento da porosidade. Além disso, os dados experimentais são mais bem representados, em termos de comportamento da relação $\mathrm{E} \mathrm{x}$ $\mathrm{P}$, para a equação D [15], apesar de o melhor ajuste ser para a equação $\mathrm{C}$. Desvios de resultados podem ser atribuídos a diferenças de processamento, geometria dos poros, arranjos cristalinos, bem como a atenuações e desvios das medidas ultrassônicas [11], não avaliadas neste estudo. O controle dimensional deu-se pela análise comparativa entre os volumes calculados a partir das dimensões iniciais e finais (diâmetro e altura interno e externo das peças cerâmicas a verde e sinterizadas). O desvio da geometria hemisférica $(\Omega)$, para as peças a verde e sinterizadas foi calculado pela equação $\mathrm{E}$ adaptada [16], em relação ao volume $\left(\mathrm{V}_{\mathrm{x}}\right)$, onde $\mathrm{x}$ é o indicativo do volume calculado pelos diâmetros (D) ou pelas alturas (h), Tabela IV. A geometria não terá desvios caso a relação $\mathrm{V}_{\mathrm{h}} / \mathrm{V}_{\mathrm{D}}$ seja igual a 1, pois, tanto os volumes calculados através da altura quanto os calculados através dos diâmetros deverão ser iguais e, portanto, $\Omega$ ser igual
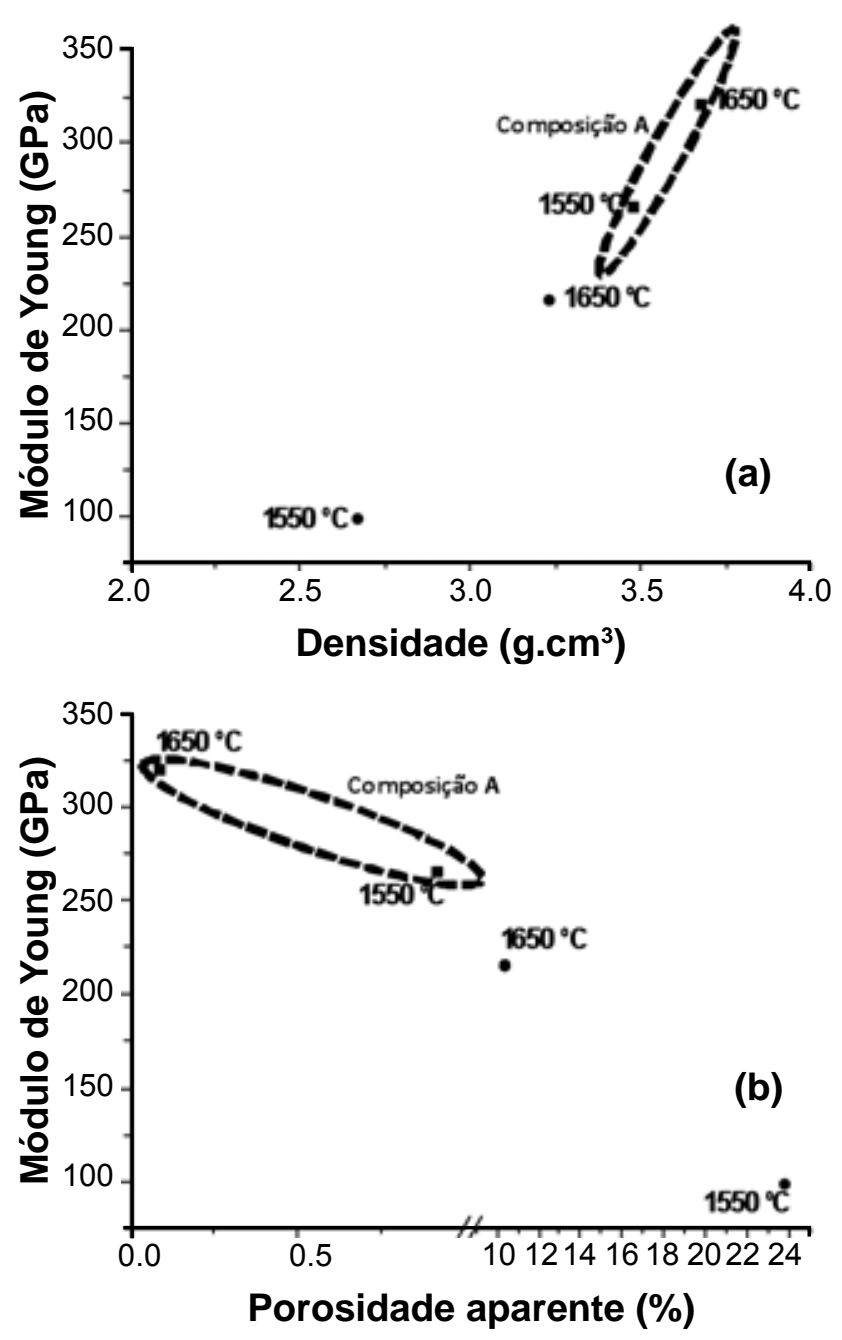

Figura 3: Módulo de Young em função da densidade e da porosidade das peças das composições A e B sinterizadas a $1550{ }^{\circ} \mathrm{C}$ e $1650{ }^{\circ} \mathrm{C}$. [Figure 3: Young modulus as a function of porosity and density parts of compositions $A$ and $B$ sintered at $1550^{\circ} \mathrm{C}$ and $1650^{\circ} \mathrm{C}$.]

a zero. Os volumes foram calculados pela equação $\mathrm{F}$, com auxílio das equações $\mathrm{G}, \mathrm{H}, \mathrm{I}$ e J.

$$
\begin{aligned}
& \Omega=\frac{\left(\mathrm{V}_{\mathrm{h}} / \mathrm{V}_{\mathrm{D}}\right)_{\text {medido }}-\left(\mathrm{V}_{\mathrm{h}} / \mathrm{V}_{\mathrm{D}}\right)_{\text {ideal }}}{\left(\mathrm{V}_{\mathrm{h}} / \mathrm{V}_{\mathrm{D}}\right)_{\text {ideal }}} \\
& \mathrm{V}_{\mathrm{x}}=2 \pi\left(\mathrm{r}_{\mathrm{e}}^{3}-\mathrm{r}_{\mathrm{i}}^{3}\right)
\end{aligned}
$$

nas quais $r_{e}$ e $r_{i}$ são os raios internos e externos representados: para o volume $\left(\mathrm{V}_{\mathrm{D}}\right)$ calculado pelos diâmetros, interno e externo, $r_{e}=D_{e}^{/} / 2$ e $r_{i}=D_{i} / 2$. Para o volume $\left(\mathrm{V}_{\mathrm{h}}\right)$ calculado pelas alturas, internas e externas, $r_{e}=h_{e}, r_{i}=h_{i}$.

Os desvios da geometria proposta (hemisférica) começaram nas peças conformadas a verde, Tabela IV. O volume interno ao hemisfério é maior do que o volume externo ao hemisfério, Fig. 1. Pode-se atribuir este fato a vários de fatores, sendo os mais pronunciados o nãotratamento das matérias-primas, que ocasionou uma mobilidade de maneira irregular da mistura no interior do 


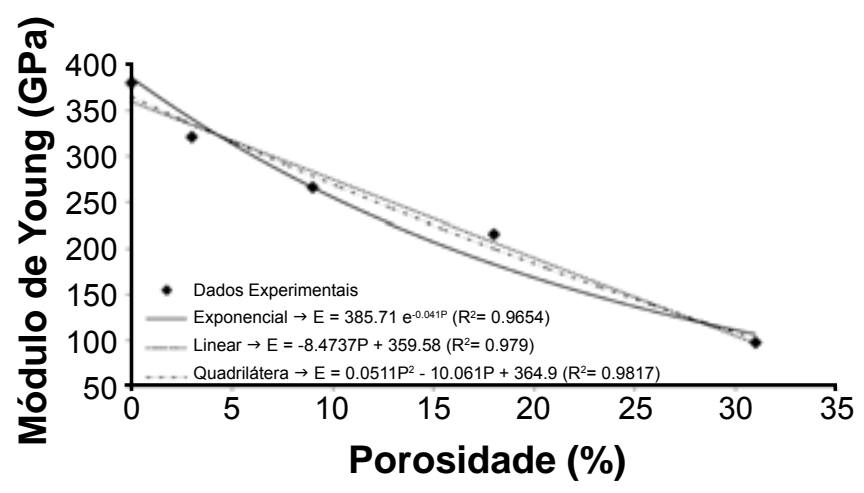

Figura 4: Comparativo entre modelos empíricos e dados experimentais.

[Figure 4: Comparison between empirical models and experimental data.]

Tabela V - Desvios da geometria hemisférica para as peças a verde e sinterizadas das composições A e B.

[Table $V$-Deviations from the hemispherical geometry for the green and sintered parts of the compositions $A$ and B.]

\begin{tabular}{cccc}
\hline \multirow{2}{*}{ Composição } & \multicolumn{3}{c}{ Desvio da geometria hemisférica $(\%)$} \\
\cline { 2 - 4 } & a verde & $1650{ }^{\circ} \mathrm{C}$ & $1550{ }^{\circ} \mathrm{C}$ \\
\hline A & 37,53 & 22,84 & 18,10 \\
B & 31,12 & 16,4 & 18,67 \\
\hline
\end{tabular}

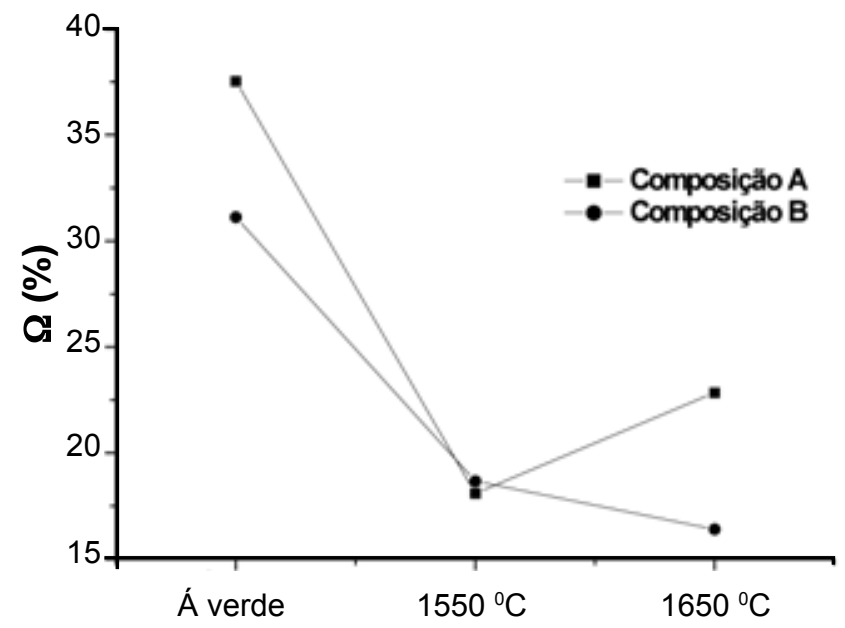

Figura 5: Desvios da geometria para as peças a verde e sinterizadas de composições A e B.

[Figure 5: Deviations of the geometry for the green and sintered parts of compositions $A$ and $B$.]

molde [17], o projeto do molde utilizado [18], que não levou em consideração o atrito acentuado entre a cerâmica e o molde, acarretando uma transmissão irregular da pressão de compactação e, por conseqüência, não permitindo uma ação efetiva do molde [19]. Além disso, o atrito entre as partículas das composições, também, influenciaram no empacotamento das composições no molde [19]. O processo de sinterização "auxiliou" na correção dos desvios de geometria hemisférica proposta para ambas as composições, em termos relativos,
Tabela IV - Porosidade relativa e módulo de Young das peças sinterizadas de composições A e B, submetidas aos ciclos térmicos I e II.

[Table IV - Relative porosity and Young's modulus of sintered parts of compositions $A$ and B, subjected to thermal cycling I and II.]

\begin{tabular}{ccccc}
\hline \multirow{2}{*}{ Composição } & \multicolumn{2}{c}{$\begin{array}{c}\text { Porosidade } \\
\text { relativa }(\%)\end{array}$} & \multicolumn{2}{c}{$\begin{array}{c}\text { Módulo de Young } \\
(\mathrm{GPa})\end{array}$} \\
\cline { 2 - 5 } & $1650{ }^{\circ} \mathrm{C}$ & $1550{ }^{\circ} \mathrm{C}$ & $1650{ }^{\circ} \mathrm{C}$ & $1550{ }^{\circ} \mathrm{C}$ \\
\hline A & 0,09 & 0,92 & 321,37 & 266,18 \\
B & 10,41 & 23,88 & 216,29 & 97,80 \\
\hline
\end{tabular}

Fig. 5, apesar de que, normalmente, esta propriedade causa sérios defeitos às peças cerâmicas como, por exemplo, empenamentos em cerâmicas de superfície plana [6]. Isto pode ser atribuído, entre outros fatores, a diferentes retrações proporcionadas pelos diferentes tipos de sinterização apresentados pelas composições e, também, devido a uma distribuição irregular da pressão de compactação ao longo da altura [20], causando uma densificação menor e, conseqüentemente, uma retração maior para esta dimensão [21]. Outro fator que pode ter contribuído para estes desvios de geometria é a qualidade do molde utilizado, pois, isto acarreta um desgaste prematuro do mesmo [18] e, conseqüentemente, uma elevação da influência do atrito entre a cerâmica e o molde e da irregularidade na transmissão da pressão de compactação ao longo do corpo-de-prova. Para a temperatura de sinterização de $1550{ }^{\circ} \mathrm{C}$ houve uma maior correção do desvio, provavelmente, devido a um maior controle das retrações sofridas pelas peças cerâmicas.

\section{CONCLUSÕES}

A prensagem uniaxial foi eficaz para a produção de peças cerâmicas a base de alumina com geometria hemisférica. Observou-se que o processo de sinterização em presença de fase líquida proporcionou cerâmicas com maiores densidades e conseqüentemente com menores porosidades. Além disso, a maior temperatura de sinterização levou a densidades maiores e a porosidades menores, pois, este processo é termicamente ativado. Não houve a identificação de contaminantes ou outras fases que pudessem causar distorções tanto para a conformação, como para a sinterização de acordo com os difratogramas obtidos, dentro do limite de detecção. Verificou-se que o modelo proposto pela equação $\mathrm{D}$, foi o que melhor representou os dados obtidos para a relação $\mathrm{E} x$ $\mathrm{P}$ utilizando medições ultrassônicas. Em termos de controle dimensional, as matérias-primas utilizadas, a distribuição da pressão de compactação e a qualidade do molde foram decisivas para o desvio da geometria proposta (hemisférica). Entretanto o processo de sinterização "auxiliou” na correção destes desvios da geometria. Cabe ressaltar, ainda, que não foram levadas em consideração as pequenas recuperações elásticas das partículas do pó posterior ao alívio da pressão de compactação, pois, esta propriedade mostrouse insignificante quando as medições realizadas no molde, 
antes e posterior a prensagem, e no corpo-de-prova a verde não apresentaram diferenças significativas, e, portanto, não havendo grandes deformações na matriz em aço SAE 1045. Porém, esta propriedade será estudada para efeito das tolerâncias da futura peça produzida a base de PZT.

\section{AGRADECIMENTOS}

À Marinha do Brasil e, em especial, ao Instituto de Pesquisas da Marinha (IPqM), pelo suporte administrativo, financeiro e técnico. Aos colegas e amigos do Grupo de Tecnologia de Materiais e Armas do IPqM pelo auxílio técnico. Aos professores e funcionários do Programa de Engenharia Metalúrgica e de Materiais, pelos ensinamentos oferecidos para que fosse possível o desenvolvimento de toda a pesquisa, em especial ao Prof. T. Ogasawara, in memoriam. Ao IME pelas análises de difração de raios $\mathrm{X}$ e ao IEN pelas inspeções ultrassônicas.

\section{REFERÊNCIAS}

[1] M. A. P. dos Santos, "Síntese e Caracterização de Cerâmicas Piezoelétricas Tipo PZT Utilizada em Transdutores Eletroacústicos a partir de Insumos Nacionais", Diss. Mestrado, PEMM-COPPE/UFRJ, Rio de Janeiro, RJ (1998).

[2] E. Ringaard, T. Wurlitzer, J. Eur. Ceram. Soc. 25 (2005) 2701.

[3] L. B. Barbosa, L. M. de Jesus, Z. S. Macedo, Scientia Plena 6 (2010) 1-5.

[4] V. A. A. Santos, E. Prestes, J. Medeiros, J. L. B. Veiga, V. C. Pandolfelli, Cerâmica 57, 342 (2011) 231-236.

[5] M. A. P. dos Santos, C. A. C. Neto, E. P. Magalhães, $45^{\circ}$ CBC, Florianópolis, SC (2001) 01-10.

[6] J. S. Reed, Introduction to the Principles of Ceramic Processing, $2^{\text {nd }}$ Ed., John Wiley \& Sons Inc., New York, EUA (1995).
[7] W. Barsoun, Fundamentals of Ceramics, Mc Graw-Hill Co., New York, EUA (1997).

[8] J. C. Rocha, "Produção de Alumina Sinterizada a partir do pó e Otimização dos Parâmetros e Sinterização para a Máxima Resistência Mecânica”, Diss. Mestrado, Instituto Militar de Engenharia, Rio de Janeiro, RJ (1981).

[9] C. G. Bergeron, S. H. Risbud, Introduction to Phase Equilibria in Ceramics, in: The Am. Ceram. Soc. Book Edition, Westerville, Ohio, EUA (2000) 115.

[10] M. Bhardwaj, N. Kulkarni, B. Moudgil, Bull. Am. Ceram. Soc. 73 (1994) 83-85.

[11] F. A. Berutti, R. P. Vieira, A. K. Alves, C. P. Bergman, Anais $17^{\circ} \mathrm{CBECiMat,} \mathrm{Foz} \mathrm{do} \mathrm{Iguaçu,} \mathrm{PR} \mathrm{(2006)} \mathrm{676-683.}$

[12] J. A. Rodrigues, F. S. Ortega, A. E. M. Paiva, E. L. G. Villaboim, V. C. Pandolfelli, Cerâmica 50, 315 (2004) 209216.

[13] H. N. Yoshimura, A. L. Molisani, G. R. Siqueira, A. C. Camargo, N. E. Narita, P. F. Cesar, H. Goldstein, Cerâmica 51, 319 (2010) 239-251.

[14] W. D. Kingery, H. K. Bowen, D. H. Uhlmann, Introduction to Ceramics, $2^{\text {nd }}$ Ed., John Wiley \& Sons, New York, EUA (1976).

[15] R. M. Spriggs, J. Am. Ceram. Soc. 44 (1962) 628-629.

[16] C. D. Rocha, M. A. P. dos Santos, C. A. C. Neto, J. M. de Melo, IX ETAS, Rio de Janeiro, RJ (2011) 82-88.

[17] R. A. Thompson, Mechanics of Powder Pressing I-III, Bull. Am. Ceram. Soc. 60 (1981) 237-245.

[18] C. D. Rocha, "Estudo do Processamento da Alumina visando Utilização em Geometria Hemisférica", Diss. Mestrado, PEMM-COPPE/UFRJ, Rio de Janeiro, RJ (2011). [19] J. L. A. Albaro, Cerâmica Ind., Parte 1 e 2 (2000) 23-28 e 14-20.

[20] N. Özkan, B. J. Bricoe, Ceram. Int. 23 (1997) 521-536. [21] D. W. Richerson, Forming and Predensification, and Non traditional Densification Processes, Eng. Mater. Handbook, 4 - Ceramics and Glasses, ASM Int., Section 1 (1991) 123.

(Rec. 21/02/2012, Rev. 27/05/2013, Ac. 03/06/2013) 